\title{
Correlation and Agreement of Steady-State Free Processed Imaging Cardiac Magnetic Resonance Imaging and Balloon Waist Diameter of the Right Ventricular Outflow Tract for Percutaneous Pulmonary Valve Replacement
}

\author{
Joshua David Kurtz, MD*, Anthony M. Hlavacek, MD, George Hamilton Baker, MD \\ Department of Pediatrics, Division of Pediatric Cardiology, Medical University of South Carolina, Charleston, South Carolina, USA
}

\begin{abstract}
Background: Percutaneous pulmonary valve replacement (PPVR) candidacy is limited by right ventricular outflow tract (RVOT) diameter.

Objective: We examined the correlation and agreement of RVOT minimal diameter measured by MRI and balloon waist diameter (BWD) during PPVR.

Methods: This is a single center, retrospective study of patients undergoing PPVR who had a cardiac MRI performed within one year prior to the procedure. AlI MRI measurements were made by a single investigator at the narrowest location of the RVOT during peak systole in two orthogonal planes using three separate MRI sequences. BWD was defined as the narrowest point in the sizing balloon at full inflation within the RVOT. The primary outcome was the agreement of MRI and BWD measurements of the RVOT.
\end{abstract}

Results: Twenty-three patients were included in the analysis. Twelve (52\%) were male, $17(74 \%)$ had a diagnosis of tetralogy of Fallot, $4(17 \%)$ did not have a valve placed due to RVOT size. The average age was 31 years (9-56 years old). BWD measurements had a significant correlation with both planes of stacked cine steadystate free precession imaging MRI and the larger diameter of MR angiography. BWD had significant agreement (c) 2019 Journal of Structural Heart Disease Published by Science International Corp. ISSN 2326-4004

Accessible online at: http://structuralheartdisease.org/ with both stacked cine steady-state free precession imaging MRI planes by Bland-Altman analysis.

Conclusions: MRI measurements show moderate correlation and agreement with BWD of the RVOT. While the mean difference is small, the range of agreement is quite wide. This suggests MRI is only moderately effective in determining RVOT diameter candidacy in PPVR. Further study is warranted to determine the most effective method for RVOT diameter selection in PPVR.

Copyright @ 2019 Science International Corp.

\section{Key Words}

Percutaneous Pulmonary valve replacement • Tetralogy of Fallot • Right ventricular outflow tract dysfunction • Pulmonary regurgitation - Cardiac magnetic resonance imaging

\section{Introduction}

Corrective surgery for patients with diseases of the pulmonary valve (PV) and right ventricular outflow tract (RVOT), has improved significantly over recent decades. Despite advances in techniques that have led to significant improvements in morbidity, mortality, and quality of life for these patients, many will require future procedures due to residual pulmonary

* Corresponding Author:

Joshua David Kurtz, MD

Division of Pediatric Cardiology

Medical University of South Carolina

165 Ashley Ave MSC 915, Charleston, SC 29425, USA

Tel.: +1 843834 0972; Fax: +1 843792 5878; E-Mail: kurtzj@musc.edu 

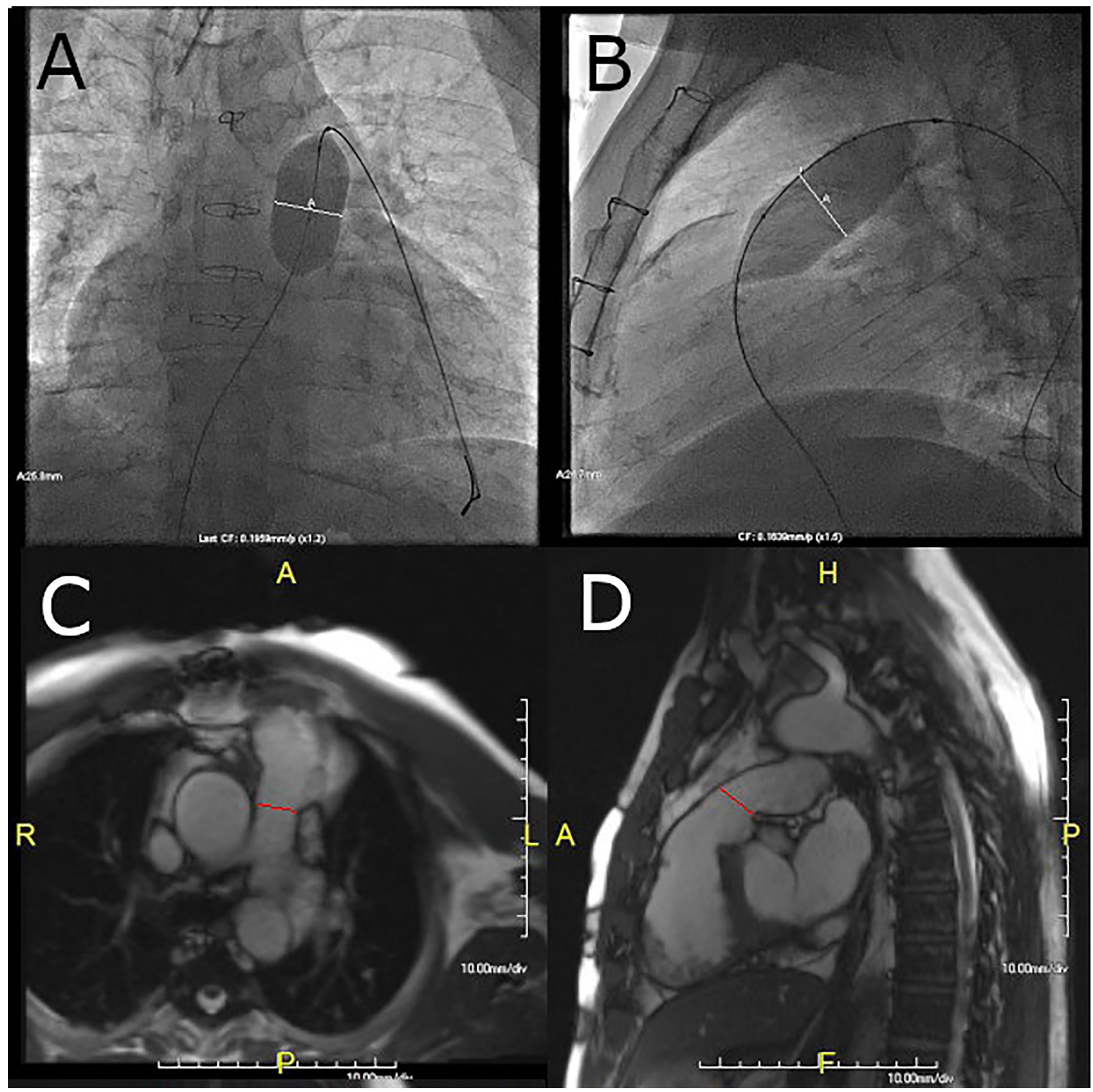

Figure 1. Examples of RVOT measurements. Panel A. BWD in AP fluoroscopy. Panel B. BWD in lateral flouroscopy. Panel C. cine steadystate free precession imaging (CSSFP) in oblique sagittal plane. Panel D. cSSFP in oblique coronal plane.

regurgitation [1]. The past two decades have seen the emergence of percutaneous valve replacement procedures. This has given new options to those patients whom have undergone surgical and catheter based procedures on their PV and RVOT. Unfortunately with the current devices, the patient population eligible for this procedure is limited by the size and shape of the outflow tract [2]. For the most part, these devices have been limited to right ventricle to pulmonary artery conduits or valve in valve replacements, with some use in native outflow tracts $[3,4]$. 
Despite the constraints to which patients are eligible for percutaneous pulmonary valve replacement (PPVR), there is still no consensus about whom to recommend for PPVR rather than surgery. The main reason for replacing the pulmonary valve in this population is the deleterious effects of pulmonary regurgitation, including arrhythmia risk, decline in right ventricular function, exercise intolerance, and risk of sudden death $[2,5]$. Cardiac MRI is considered to be the gold standard in evaluating right ventricular size and function in patients with repaired RVOT lesions. Cardiac MRI is also considered the most reliable modality in clinical decision making regarding timing of pulmonary valve replacement; thus many of these patients will undergo this procedure as part of routine management $[5,6]$. However, there are few studies evaluating the effectiveness of cardiac MRI as a screening tool for PPVR candidacy and no studies to our knowledge that evaluated the ability of cardiac MRI to determine candidacy based on RVOT size.

Balloon waist diameter (BWD) is still the gold standard measurement that determines if a PPVR can be performed, but this requires an invasive procedure. While the risk of diagnostic catheterization is low, it is not negligible [7]. The aim of this study is to determine the correlation and agreement of the RVOT minimal diameter between pre-procedural cardiac MRI and the measured BWD of the RVOT in patients undergoing attempted or successful PPVR.

\section{Methods}

This was a single center, retrospective study of patients undergoing PPVR who had a cardiac MRI performed within one year prior to the procedure. The Institutional Review Board at the Medical University of South Carolina approved the project. All patients who underwent catheterization for attempted PPVR were eligible. Patients were excluded if they did not have a cardiac MRI within one year prior to the catheterization at this institution, the PPVR was done via perventricular technique, or BWD measurements were not available.

The cardiac MRI studies were performed using a 1.5 T system (Magnetom Avanto, Siemens Healthineers, Erlangen, Germany), following a standard institutional clinical protocol. Measurements were made from three different MRI sequences: stacked cine steadystate free-precession imaging (cSSFP) through the RVOT in two planes, contrast-enhanced magnetic resonance angiography (MRA), and a self-navigated three-dimensional steady-state free-precession (3D-SSFP) technique. The cSSFP planes were selected for measurement from stacked "4-chamber" slices (oblique coronal plane) and slices taken through the RVOT perpendicular to the " 4 chamber" view (oblique sagittal plane). The MRA and 3D-SSFP images were uploaded to a separate workstation for 3D reconstruction (Aquarius iNtuition, TeraRecon, San Mateo, Calif.) Cardiac cycle was gated for CSSFP and 3D-SSFP protocols. cSSFP images were retrospectively gated with 25 phases per cardiac cycle taken during an expiratory breath hold. The field of view was adjusted for body size. All cardiac MRI measurements were performed by a single investigator (JDK). Measurements from cSSFP images were made during peak systole at the narrowest diameter of the RVOT (Figure 1). MRA and 3D-SSFP measurements were made of the cross-sectional diameter at the narrowest portion of the RVOT. Two measurements of the cross sectional diameter were taken perpendicular to each other. BWD was measured at the time of catheterization and was defined as the narrowest point in the sizing balloon at full inflation within the RVOT (Figure 1). This was done using a compliant sizing balloon, with few exceptions Amplatzer ${ }^{\mathrm{TM}}$ sizing balloon II (St. Jude, St. Paul, MN), which was expanded using hand inflation until a waist was seen in the RVOT. Volumetric data was extracted from cardiac MRI reports.

Categorical variables are described as counts and percentages; continuous variables are described using means and standard deviations. Measurements of the BWD diameter in the anteroposterior (AP) and lateral views were plotted against the cardiac MRI measurements in each protocol. Correlation analysis was done using Pearson's " $r$ ". Agreement was detected using Bland-Altman plots.

\section{Results}

There were 23 patients who met inclusion criteria. Of those, 18 (78\%) had BWD measurements available in both AP and lateral orientation, 22 (96\%) had lateral BWD measurements available for comparison. 
Table 1: Demographic Data

\begin{tabular}{|c|c|}
\hline Category & Count (\%) \\
\hline Age & $31.3(9-56)$ \\
\hline \multicolumn{2}{|l|}{ Sex } \\
\hline Male & $12(52 \%)$ \\
\hline Female & $11(48 \%)$ \\
\hline \multicolumn{2}{|l|}{ Diagnosis } \\
\hline Tetralogy of Fallot & $17(74 \%)$ \\
\hline Pulmonary stenosis & $2(9 \%)$ \\
\hline PA/IVS & $1(4 \%)$ \\
\hline Pulmonary regurgitation & $1(4 \%)$ \\
\hline Truncus Arteriousus & $1(4 \%)$ \\
\hline Rheumatic heart disease & $1(4 \%)$ \\
\hline \multicolumn{2}{|l|}{ Initial Surgery } \\
\hline Transannular patch & $12(52 \%)$ \\
\hline RV-PA conduit & $5(22 \%)$ \\
\hline Pulmonary valvotomy & $2(9 \%)$ \\
\hline Ross & $1(4 \%)$ \\
\hline Valve sparing repair & $1(4 \%)$ \\
\hline Not known & $1(4 \%)$ \\
\hline None & $1(4 \%)$ \\
\hline \multicolumn{2}{|l|}{ Valve Placed } \\
\hline Yes & $17(74 \%)$ \\
\hline No & $6(26 \%)$ \\
\hline \multicolumn{2}{|c|}{ Reason for unsuccessful placement } \\
\hline RVOT size & $4(67 \%)$ \\
\hline Coronary compression & $1(17 \%)$ \\
\hline Improvement with angioplasty & $1(17 \%)$ \\
\hline \multicolumn{2}{|l|}{ Valve Type } \\
\hline Melody & $14(82 \%)$ \\
\hline Sapien & $3(18 \%)$ \\
\hline
\end{tabular}

Categorical variables expressed as count (\%) Continuous variables expressed as mean (range). PA/IVS = Pulmonary atresia with intact ventricular septum; $\mathrm{RV}-\mathrm{PA}=$ Right ventricle to pulmonary artery; RVOT = Right ventricular outflow tract

Seventeen (74\%) patients had a valve placed successfully, four (17\%) were not placed due to RVOT size. Fourteen (82\%) of the 17 successfully placed valves were Melody ${ }^{\mathrm{TM}}$ valves (Medtronic Inc, Minneapolis, $M N)$, the other $3(18 \%)$ were Sapien valves (Edwards

Kurtz J. D. et al.
Table 2: Baseline Measurement and Volumetric Data

\begin{tabular}{ll}
\hline & Mean (SD) \\
\hline RVOT Measurements (mm) & \\
BWD in AP plane & $20.9(4.5)$ \\
BWD in lateral Plane & $20.9(5.1)$ \\
cSSFP coronal plane & $20.9(4.1)$ \\
cSSFP sagittal plane & $20.5(4.7)$ \\
MRA larger diameter & $25.6(4.5)$ \\
MRA smaller diameter & $19.4(5.0)$ \\
3D-SSFP MRI larger diameter & $24.2(4.1)$ \\
3D-SSFP MRI smaller diameter & $19.5(3.9)$ \\
MPA Measurements (mm) & \\
cSSFP coronal plane & $26.0(4.0)$ \\
CSSFP sagittal plane & $24.8(6)$. \\
MRA larger diameter & $29.5(6.9)$ \\
MRA smaller diameter & $24.0(6.1)$ \\
3D-SSFP MRI larger diameter & $29.6(7.1)$ \\
3D-SSFP MRI smaller diameter & $23.3(4.8)$ \\
Right ventricular end-diastolic volume & $118.8(39.6)$ \\
(ml/m²) & $66.4(29.3)$ \\
Right ventricular end-systolic volume & \\
(ml/m²) & $47.8(9.4)$ \\
Right ventricular ejection fraction (\%) & $32.3(17.1)$ \\
Pulmonary regurgitant fraction (\%) & \\
\hline
\end{tabular}

Variables are expressed as mean (SD). BWD = Balloon waist diameter; $\mathrm{AP}=$ Anterior-posterior fluoroscopy; cSSFP = stacked cine steady-state free precession imaging; MRA = Magnetic resonance angiography; 3D-SSFP = whole heart self-navigated radial MRI

Life Science, Irvine, $\mathrm{Ca}$ ). The average age at the time of the procedure was 31.4 years-old (9-56) and 12 (52\%) were male. Tetralogy of Fallot was the most common lesion ( $n=17,74 \%)$, the other diagnoses included pulmonary stenosis $(n=2,9 \%)$ and one each of truncus arteriosus, ventricular septal defect with pulmonary regurgitation, pulmonary atresia with intact ventricular septum, and rheumatic heart disease $(n=4,16 \%)$. Table 1 contains full demographic data.

The average RVOT measurement was $20.9 \pm 4.5$ $\mathrm{mm}$ by BWD in the AP plane and $20.9 \pm 5.1 \mathrm{~mm}$ in the lateral plane. The RVOT average measurement by cSSFP was $20.9 \pm 4.1 \mathrm{~mm}$ in the coronal plane and $20.5 \pm$ $4.7 \mathrm{~mm}$ in the sagittal plane. The main pulmonary ar- 

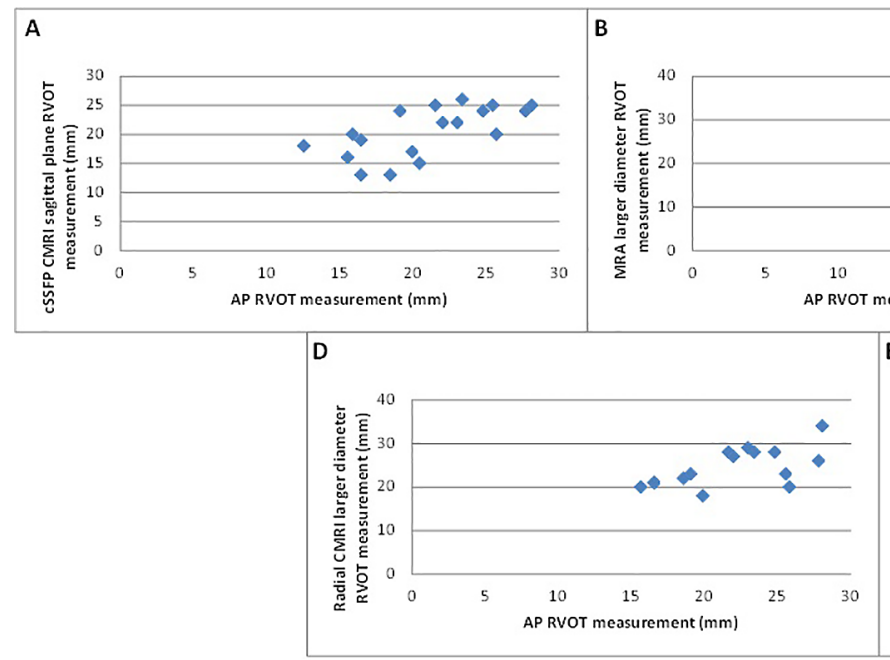
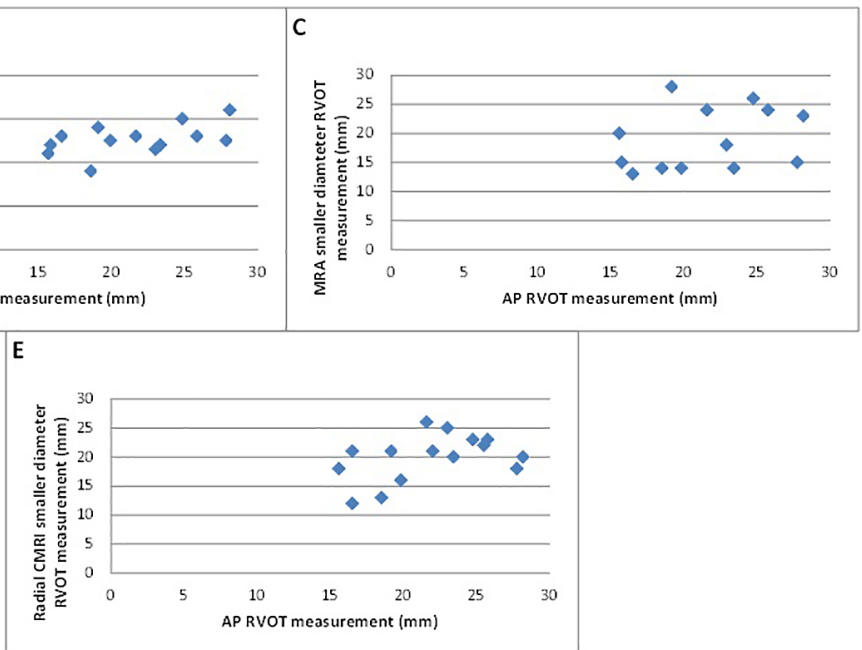

Figure 2. Comparison of anteroposterior angiogram and cardiac MRI measurements of the RVOT. Scatter plot comparison of anteroposterior angiogram versus; Panel A. cSSFP coronal plane, $r=0.65$, Panel B. MRA larger diameter, $r=0.51$, Panel C. MRA smaller diameter, $r=0.30$, Panel D. 3D-SSFP, $r=0.62$, Panel E. 3D-SSFP smaller diameter, $r=0.43$.
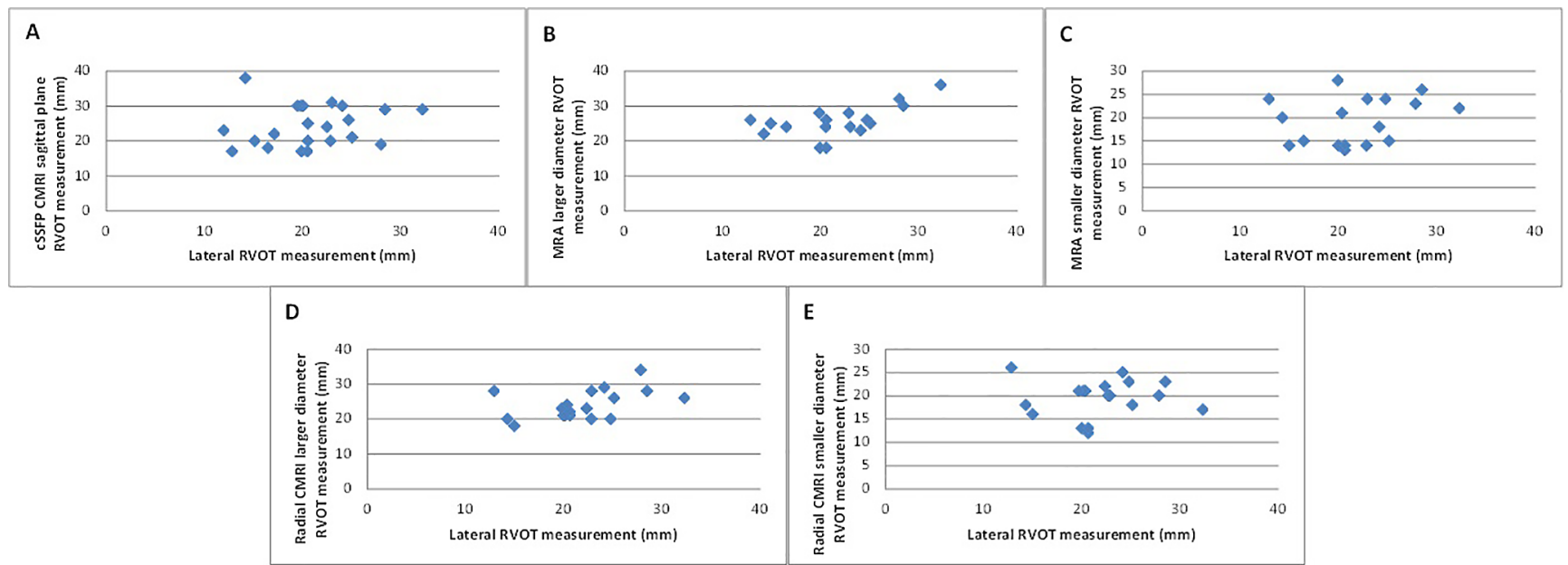

Figure 3. Comparison of lateral angiogram and cardiac MRI measurements of the RVOT. Scatter plot comparison of lateral angiogram versus; Panel A. cSSFP sagittal plane, $r=0.67$, Panel B. MRA larger diameter, $r=0.60$, Panel C. MRA smaller diameter, $r=0.25$, Panel $D$. 3D-SSFP larger diameter, $r=0.48$, Panel E. 3D-SSFP smaller diameter, $r=0.06$.

tery (MPA) average measurement was $26.0 \pm 4.0 \mathrm{~mm}$ and $24.8 \pm 6.0 \mathrm{~mm}$ for coronal and sagittal planes respectively. MRA and 3D-SSFP images were obtained in 18 and 19 patients, respectively. The average RVOT measurement by MRA was $25.6 \pm 4.5 \mathrm{~mm}$ for the larger diameter and $19.4 \pm 5.0 \mathrm{~mm}$ for the smaller diameter. The MPA was $29.5 \pm 6.9 \mathrm{~mm}$ for the larger diameter and $24.0 \pm 6.1 \mathrm{~mm}$ for the smaller diameter. By 3D-SSFP imaging, the RVOT average measurement was $24.2 \pm 4.1 \mathrm{~mm}$ for the larger diameter and $19.5 \pm$ $3.9 \mathrm{~mm}$ for the smaller diameter; the MPA was $29.6 \pm$
$7.1 \mathrm{~mm}$ for the larger diameter and $23.3 \pm 4.8 \mathrm{~mm}$ for the smaller diameter. Volumetric data for the cohort revealed an average indexed right ventricular end diastolic volume was $118.8 \pm 39.6 \mathrm{~mL} / \mathrm{m}^{2}$, average right ventricular ejection fraction of $47.8 \pm 9.4 \%$, and a pulmonary regurgitant fraction of $32.3 \pm 17.1 \%$. See Table 2 for full measurements and volumetric data.

Balloon waist diameter measurements were plotted against cardiac MRI measurements to determine correlation. BWD in the AP view (Figure 2) was compared to coronal plane of cSSFP images $(r=0.65, p<$ 


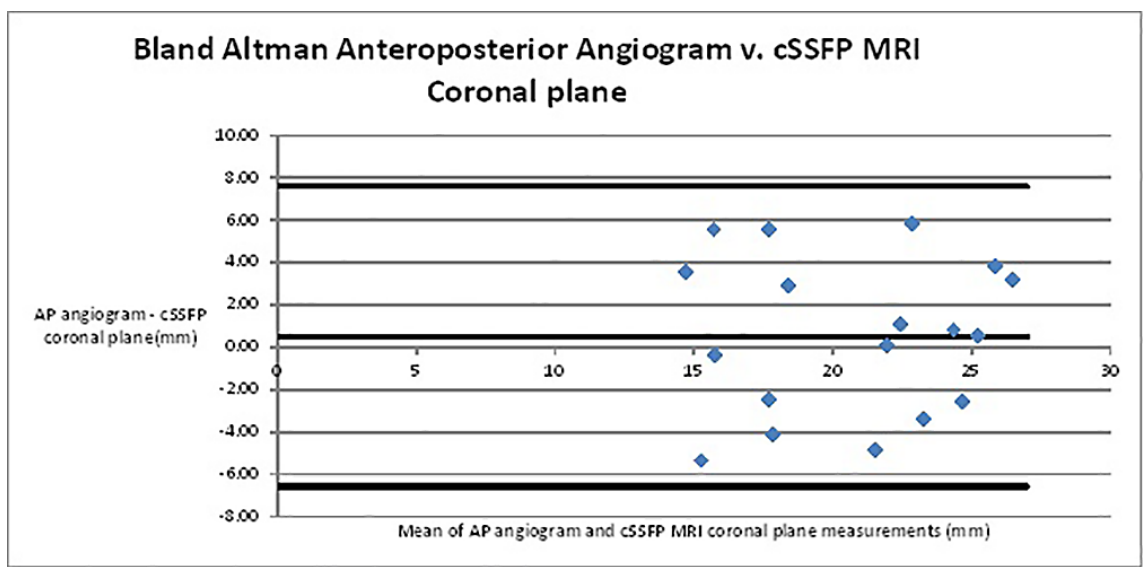

Figure 4. Bland Altman plot of anteroposterior angiogram versus CSSFP MRI in coronal plane.

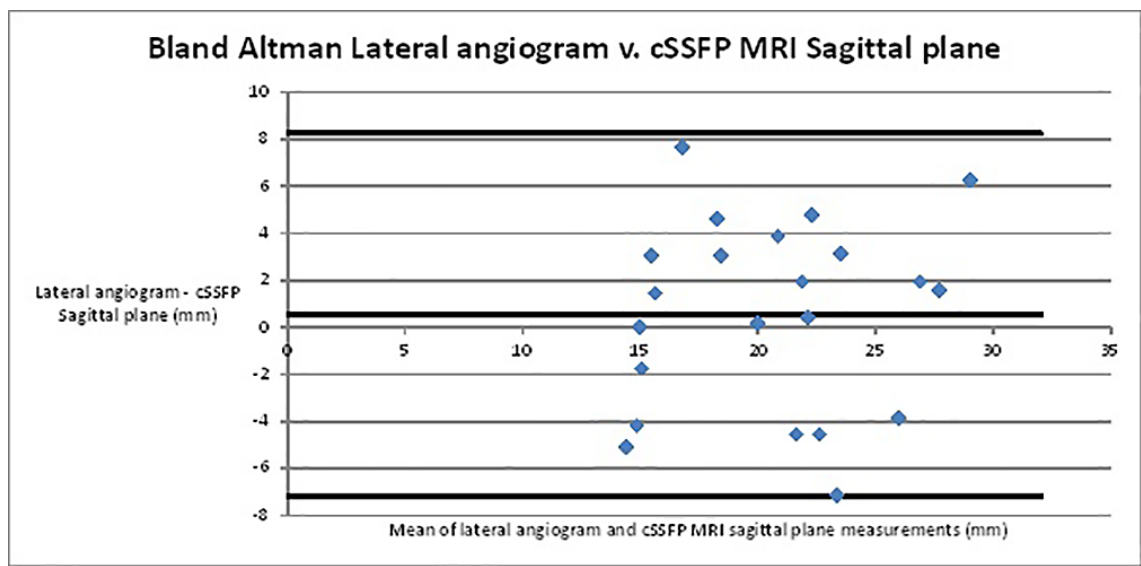

Figure 5. Bland Altman plot of lateral angiogram versus CSSFP MRI in sagittal plane.

0.01), MRA larger diameter $(r=0.51, p=0.07)$, MRA smaller diameter $(r=0.30, p=0.3)$, 3D-SSFP larger diameter $(r=0.62, p=0.01)$, and 3D-SSFP smaller diameter $(r=0.43, p=0.1)$. BWD in the lateral view (Figure 3 ) was compared to sagittal plane of cSSFP images $(r=0.67, p<0.01)$, MRA larger diameter $(r=0.60, p=0.01)$, MRA smaller diameter $(r=0.25$, $p=0.4)$, 3D-SSFP larger diameter $(r=0.48, p=0.06)$, and 3D-SSFP smaller diameter $(r=0.06, p=1.0)$. CSSFP measurements showed the strongest correlation and underwent Bland-Altman analysis for agreement (Figures 4 and 5). Bland-Altman analysis of BWD in the AP view compared to the CSSFP coronal plane showed a mean measurement difference of $0.50 \pm$ $3.62 \mathrm{~mm}(95 \% \mathrm{Cl}-6.6-7.6)$. Bland-Altman analysis of BWD in the lateral view compared to the CSSFP sagittal plane showed a mean measurement difference of $0.54 \pm 3.94 \mathrm{~mm}(95 \% \mathrm{Cl}-7.2-8.3)$.

\section{Discussion}

The present study's results suggest that cardiac MRI can be a useful tool to predict which patients will be a candidate for PPVR based on RVOT size. To our knowledge, this is the first study to examine the ability of cardiac MRI to be used as a tool for PPVR candidate selection. The mean difference between the BWD and cSSFP measurements of the RVOT was only $\sim 0.5 \mathrm{~mm}$; however, the standard error around this point was wide. This indicates cardiac MRI only has a moderate capability to predict if percutaneous pulmonary valve implantation will be successful in patients with borderline RVOT size.

One difficulty with cardiac MRI's ability to predict which candidates will be eligible based on RVOT size is distensibility. While cardiac MRI can account for some distensibilty and changes in size and shape of 
the RVOT, it is still difficult to predict the exact relationship of final stent diameter and ultimate RVOT size. This study attempted to account for this as much as possible by measuring the RVOT during peak systole on cSSFP imaging. Presumably during this time the RVOT would be expanded to its widest point. This most likely explains why cSSFP images showed the best correlation and agreement with BWD. MRA is not gated to the cardiac cycle and the measurements became an average of systole and diastole which would limit the utility in using this protocol. 3D-SSFP is gated to the cardiac cycle, but is taken at a single phase when the heart is most quiescent which may not be peak systole, thus it is not possible to know if these measurements were at the time when the RVOT was largest.

Currently, there is a significant amount of research being done into reliably predicting the distensibility within an artery $[3,8]$ A number of different compliant materials at different thicknesses have been tested to make in-vitro models of arteries in an attempt to be able to predict how distensible a vessel is non-invasively. These models are created using advanced images, mostly MRI to obtain the in-vitro data [8]. While this method is extremely expensive and time consuming, the theory behind these models is that MRI can be used to predict the characteristics of the vessel. Thus there is data to suggest that cardiac MRI can be used to non-invasively determine true RVOT size, especially when using CSSFP imaging during peak systole, as our study did [1,8].

In addition to the size of the outflow tract, the shape and character of the outflow tract can play an important role in the ability to place a percutaneous valve. Schievano et al. defined five distinct types of RVOT morphology in patients whom had undergone surgical repair of congenital heart disease affecting the RVOT. Type 1 morphology was the only morphology not amenable to PPVR. However, this type accounted for $44.6 \%$ of the patients in the Schievano study. This suggests nearly half of patients are ineligible for current PPVR based on shape of the RVOT alone [1]. The frequent large size and this pyramidal morphology of many native RVOTs after surgery have led to the use of a new self-expanding stent PPVR. This technology has shown promise in early feasibility studies and looks encouraging as an approach that will allow many more patients to receive a percutaneous valve [4, 9-11]. While this technology is still being developed and tested there have been new techniques for using the current PPVR including implanting valves in the branch pulmonary arteries [12]. Cardiac MRI will continue to be useful for both of these techniques and future studies should determine if cardiac MRI can predict an accurate cross sectional area for the safe deployment of self-expanding stents and current PPVR in the branch pulmonary arteries.

ECG gated CTA may better assess RVOT size and shape, but it is generally not utilized to follow right ventricular function and volumes over time, plus serial $C T$ exposes patients to unnecessary radiation. However, cardiac MRI is an ideal test to attempt to predict the candidacy for PPVR because most of these patients will undergo cardiac MRI as part of their standard clinical evaluation prior to consideration for valve replacement. This is due to the fact that many of the guidelines for pulmonary valve replacement are based upon severity of pulmonary insufficiency and right ventricular volume on cardiac MRI. Additionally, cardiac MRI can be used to screen for another common cause of PPVR placement failure, coronary compression [3, 5]. Malone, et al recently showed that pre-procedural screening with cardiac MRI or CTA can reliably predict which candidates are at risk for coronary compression [5].

This study shows promising results that cardiac MRI has benefit in patient screening for PPVR, but there were a number of limitations to this study. The retrospective design does not allow for determining eligibility by measurements on the cardiac MRI at the time of catheterization. Additionally, the patient population was biased by the fact that only those who were deemed to be good candidates were recommended for PPVR attempt, this decision may have included the pre-procedural cardiac MRI measurements. This study could have been improved if cardiac MRI measurements were categorized prior to catheterization as suitable for PPVR or not. Going forward a larger, multi-center study, should be undertaken to determine if these results are true amongst a larger population. A larger population could improve the uncertainty caused by wide lines of agreement and be used to create a regression formula to translate a cardiac MRI RVOT measurement to BWD measure- 
ment. This would further improve the predictive value of cardiac MRI for PPVR candidacy.

\section{Conclusion}

cSFFP MRI measurements show moderate correlation and agreement with BWD of the RVOT. While the mean difference is low, the lines of agreement are quite wide. This suggests MRI is only moderately effective in determining RVOT diameter candidacy in PPVR. Further study using larger patient samples is warranted to determine if MRI is an effective method to prospectively assess RVOT candidacy for PPVR and to determine the most effective method to screen for PPVR candidacy based on RVOT diameter.

\section{Conflict of Interest}

The authors have no conflict of interest relevant to this publication.

\section{Comment on this Article or Ask a Question}

\section{References}

1. Schievano S, Coats L, Migliavacca F, Norman W, Frigiola A, Deanfield J, et al. Variations in Right Ventricular Outflow Tract Morphology Following Repair of Congenital Heart Disease: Implications for Percutaneous Pulmonary Valve Implantation. J Cardiovasc Magn Reson. 2007;9:687-695. PMID: 17578725

2. Phillips $A B$, Nevin $P$, Shah $A$, Olshove $V$, Garg R, Zahn EM. Development of a Novel Hybrid Strategy for Transcatheter Pulmonary Valve Placement in Patients Following Transannular Patch Repair of Tetralogy of Fallot. Catheterization and Cardiovascular Interventions. J Cardiovasc Magn Reson. 2016;87:403-410. DOI: 10.1002/ccd.26315

3. Lurz P, Bonhoeffer P, Taylor AM. Percutaneous Pulmonary Valve Implantation: An Update. Expert Rev Cardiovasc Ther. 2009;7:823-833. DOI: 10.1586/erc.09.57

4. Zahn EM, Chang JC, Armer D, Garg R. First human implant of the Alterra Adaptive Prestent(TM) : A new self-expanding device designed to remodel the right ventricular outflow tract. Catheter Cardiovasc Interv. 2018;91:1125-1129. DOI: 10.1002/ ccd. 27581

5. Malone L, Fonseca B, Fagan T, et al. Preprocedural Risk Assessment Prior to PPVI with CMR and Cardiac CT. Pediatric cardiology. 2017;38:746-753. DOI: 10.1007/s00246017-1574-0

6. Selly JB, Iriart $X$, Roubertie $F$, Mauriat $P$,
Marek J, Guilhon E, et al. Multivariable Assessment of the Right Ventricle by Echocardiography in Patients With Repaired Tetralogy of Fallot Undergoing Pulmonary Valve Replacement: A Comparative Study With Magnetic Resonance Imaging. Arch Cardiovasc Dis. 2015;108:5-15. DOI: 10.1016/j.acvd.2014.07.054

7. Stefanescu Schmidt AC, Armstrong A, Kennedy KF, Nykanen D, Aboulhosn J, Bhatt AB. Prediction of Adverse Events After Catheter-Based Procedures in Adolescents and Adults With Congenital Heart Disease in the IMPACT Registry. Eur Heart J. 2017;38:2070-2077. DOI: 10.1093/eurheartj/ehx200

8. Biglino $G$, Verschueren $P$, Zegels R, TayIor AM, Schievano S. Rapid Prototyping Compliant Arterial Phantoms for In-Vitro Studies and Device Testing. Journal of Cardiovascular Magnetic Resonance. J Cardiovasc Magn Reson. 2013;15:2. DOI: 10.1186/1532-429x-15-2

9. Gillespie MJ, Benson LN, Bergersen L, Bacha EA, Cheatham SL, Crean AM, et al. Patient Selection Process for the Harmony Transcatheter Pulmonary Valve Early Feasibility Study. Am J Cardiol. 2017;120:13871392. DOI: 10.1016/j.amjcard.2017.07.034

10. Bergersen L, Benson LN, Gillespie MJ, Cheatham SL, Crean AM, Hor KN, et al. Harmony Feasibility Trial: Acute and ShortTerm Outcomes With a Self-Expanding
Transcatheter Pulmonary Valve. JACC Cardiovascular interventions 2017;10:176373. DOI: 10.1016/j.jcin.2017.05.034

11. Garay F, Pan X, Zhang YJ, Wang C, Springmuller D. Early Experience With the Venus Pvalve for Percutaneous Pulmonary Valve Implantation in Native Outflow Tract. Neth Heart J. 2017;25:76-81. DOI: 10.1007/ s12471-016-0932-5

12. Qureshi AM, Bansal N, McElhinney DB, Boudjemline $\mathrm{Y}$, Forbes TJ, Maschietto N, et al. Branch Pulmonary Artery Valve Implantation Reduces Pulmonary Regurgitation and Improves Right Ventricular Size/ Function in Patients With Large Right Ventricular Outflow Tracts. JACC Cardiovasc Interv. 2018;11:541-550. DOI: 10.1016/j. jcin.2018.01.278

Cite this article as: Kurtz JD, Hlavacek AM, Baker GH. Correlation and Agreement of Steady-State Free Processed Imaging Cardiac Magnetic Resonance Imaging and Balloon Waist Diameter of the Right Ventricular Outflow Tract for Percutaneous Pulmonary Valve Replacement. Structural Heart Disease. 2019;5(3):62-69. DOI: https://doi. org/10.12945/j.jshd.2019.028.18 